\section{Re: Neoadjuvant Intraperitoneal and Systemic Chemotherapy for Gastric Cancer Patients with Peritoneal Dissemination}

\section{TO THE EDITORS:}

We read the article by Fujiwara et al. in the May issue of Annals of Surgical Oncology with interest. ${ }^{1}$ They analyzed a cohort of 25 gastric cancer patients with positive cytology or with peritoneal carcinomatosis who received neoadjuvant intraperitoneal and systemic chemotherapy (NIPS). The authors asserted that this new approach was highly effective and well tolerated in patients with advanced gastric cancer and peritoneal dissemination.

Gastric resection in combination with preoperative chemotherapy followed by intraperitoneal chemotherapy may be a good approach. However, we worry about possible confusion caused by unclear terminology when discussing chemotherapy. Many researchers misuse or confuse the terms neoadjuvant or preoperative to define the chemotherapy. We think that the terminology should be used properly to clarify the main object of the study.

Neoadjuvant chemotherapy is usually defined as the application of the chemotherapeutic agent as a primary treatment in patients with locally advanced cancer. The primary goal of neoadjuvant chemotherapy at this stage is to reduce the size of the primary tumor so that the surgeon can expect a better chance for a complete resection and an improvement in the overall survival rates in patients with locally advanced cancer. In our opinion, an advanced gastric cancer with synchronous distant metastasis, including peritoneal seeding, is not a candidate for neoadjuvant chemotherapy. Rather, it should be classified as palliative chemotherapy.
On the basis of several studies, the current treatment strategy for patients with P0, CY1 disease is systemic chemotherapy unless the patients have symptoms requiring palliative surgical intervention. ${ }^{2-4}$ Recent studies have revealed two important points. First, systemic fluorouraciland cisplatin-based chemotherapy alone can eliminate the free peritoneal cancer cells in a high proportion of disease with positive cytology (CY1) without any evidence of gross M1 disease (37-70\%). ${ }^{3,4}$ Second, successful clearing of CY1 greatly improves patient prognosis.

Fujiwara et al. should provide the difference of survival rates between the NIPS group and conventional systemic chemotherapy group to support their hypothesis. Their conclusion that NIPS is highly effective lacks the power of persuasion because it is not supported by comparative data.

Jung Ho Shim, MD, Cho Hyun Park, MD, PhD, and Kyo Young Song, MD, PhD

Department of Surgery, Seoul St. Mary's Hospital, College of Medicine, The Catholic University of Korea, Seoul, Korea

e-mail:skygs@catholic.ac.kr

Published Online: 23 August 2011

(C) Society of Surgical Oncology 2011

\section{REFERENCES}

1. Fujiwara Y, Takiguchi S, Nakajima K, et al. Neoadjuvant intraperitoneal and systemic chemotherapy for gastric cancer patients with peritoneal dissemination. Ann Surg Oncol. 2011;17:1770-8.

2. Mezhir JJ, Shah MA, Jacks LM, et al. Positive peritoneal cytology in patients with gastric cancer: natural history and outcome of 291 patients. Ann Surg Oncol. 2010;17:3173-80.

3. Lorenzen S, Panzram B, Rosenberg R, et al. Prognostic significance of free peritoneal tumor cells in the peritoneal cavity before and after neoadjuvant chemotherapy in patients with gastric carcinoma undergoing potentially curative resection. Ann Surg Oncol. 2010;17:2733-9.

4. Okabe H, Ueda S, Obama K, et al. Induction chemotherapy with S-1 plus cisplatin followed by surgery for treatment of gastric cancer with peritoneal dissemination. Ann Surg Oncol. 2009;16:3227-36. 